\title{
Urike Vollmer Einen Spiegel finden - Erlösung in den Filmen von Jane Campion ${ }^{1}$
}

Die neuseeländische Regisseurin Jane Campion, besonders bekannt durch ihren Film „Das Piano“, äußerte in einem Interview den Gedanken, alle ihre filmischen Hauptpersonen strebten nach Erlösung. ${ }^{2}$ Demnach geben Campions Filme im gesamten und der in ihnen jeweils erreichte, vorläufige Endpunkt der charakterlichen Entwicklung der Hauptfigur im besonderen einen Einblick in Campions Verständnis von Erlösung. Der folgende Artikel möchte Campions Auffassung von Erlösung erläutern anhand dessen, was die neuseeländische Schriftstellerin Janet Frame im Film „An Angel at My Table“ (1990), was die stumme Ada in „The Piano" (1993) und was die Amerikanerin Isabel in "The Portrait of a Lady" (1996) am Ende ihrer Entwicklung jeweils erreichen. ${ }^{3}$

Jane Campion greift in den drei Filmen den Spiegel als Metapher auf, um deutlich zu machen, dass ihre Film-Charaktere nur durch andere Menschen zu ihrem persönlichen Ziel finden. Wie bei einem Spiegelbild können die Charaktere sich nur selbst sehen, wenn sie zu einem Gegenüber in Beziehung sind, das ihnen ihr Wesen spiegelt. In Beziehung zu sein spielt sich in Campions Filmen auf drei Ebenen ab, ähnlich wie in der Theologie, wie zu zeigen sein wird.

\section{Der Tiefpunkt wird zum Wendepunkt - Gedanken zu „An Angel at My Table"}

Der Film „An Angel at My Table“ beschreibt den problematischen Selbstwerdungsprozess der neuseeländischen Schriftstellerin Janet Frame als

1 Der Artikel basiert auf meiner im Rahmen eines Master of Arts-Studienganges an der Universität Leeds geschriebenen Arbeit mit dem Titel „Taking Another Way: Grace and Redemption in the Films of Jane Campion", Leeds 1999. Die Arbeit wurde von Dr. Hugh Pyper und Gaye Ortiz betreut.

2 Vgl. Van den Berk, Tjeu, The Piano. Boeiende klanken, Kampen 1994, 9.

3 Zum Thema "Erlösung" im Film siehe auch: Kirsner, Inge, Erlösung im Film: praktisch-theologische Analysen und Interpretationen, Stuttgart 1996.

Das Buch bietet neben vier Filminterpretationen eine Verhältnisbestimmung von Film und Theologie, sowie einige eher allgemein gehaltene theologische Reflexionen zum Medium Film. Der zugrunde gelegte Begriff von Erlösung wird allerdings nur ungenau festgelegt und ein Vergleich des Erlösungsverständ̀nisses der Filme mit der Theologie findet kaum statt. Aus diesem Grund scheint der Titel des Buches nicht gerechtfertigt. 
"universale Leidensgeschichte". 4 Janet Frame, die sich aus Unsicherheit im Lauf ihrer Pubertät und als junge Frau immer mehr vom Kontakt mit ihrer Umwelt in eine fantastische Welt der Literatur und Poesie flüchtet, wird nach einem Selbstmordversuch fälschlicherweise als schizophren diagnostiziert. Janet begibt sich auf Anraten einer Ärztin in ein psychiatrisches Krankenhaus, wo sie, wie sie aus dem Off erzählt, mehr als 200 Elektroschockbehandlungen erhält, die jeweils soviel Angst wie eine Exekution auslösen. Den schwierigsten Punkt ihrer Leidensgeschichte erreicht Janet als sie hört, dass sie einer Leukotomie unterzogen werden soll, einer Gehirnoperation mit persönlichkeitsveränderndem Effekt. Die darauf folgende Szene des Films interpretiert Janets Tiefpunkt gleichzeitig als Wendepunkt ihres Leidens. Janet ist in einem kleinen Raum eingeschlossen, in dem die Tür in fast unerreichbarer Höhe angebracht ist bildlich auswegloser ist ihre Lage nie gewesen. In dieser Zelle kritzelt Janet verzweifelt ein aus ihrer Erinnerung auftauchendes Shakespearezitat an die Wand: „Fear no more the heat o' the sun, nor the furious winter's rages. Thou thy worldly task hast done." (Fürchte nicht länger der Sonne Glühen, noch des wilden Winters Wüten. Du hast deine Aufgabe in der Welt vollbracht.5) - die ersten Zeilen eines Begräbnisgesanges für Imogen in Shakespeares Drama Cymbeline. Durch dieses Zitat wird Janets Situation angesichts einer persönlichkeitsverändernden Operation mit Tod in Verbindung gebracht. Liest man das Zitat aber in seinem ursprünglichen Zusammenhang in Shakespeares Stück, so zeigt es gleichzeitig einen Neuanfang an. Die im Lied besungene Imogen erscheint nur tot als Resultat eines eingenommenen Schlafmittels. Ihr Wiedererwachen markiert eine Veränderung ihrer Identität und auf diese Weise beschreibt Shakespeare Imogens charakterliche Verwandlung symbolisch als Tod- und Auferstehungsgeschehen. Durch die Verwendung des Zitats wird also auch im Film nicht nur ein symbolischer Tod, sondern auch eine symbolische Auferstehung impliziert. Der persönliche Tiefpunkt ist gleichzeitig Wendepunkt, weil die vorher von menschlichen Kontakten isolierte Janet zu einer menschlichen Verbindung mit Shakespeares Imogen und auf diese Weise zur Hoffnung auf ein „Weiterleben“ nach ihrem symbolischen Tod findet. Janets Erkenntnis, dass sie sich in Todesnähe befindet wird gleichzeitig zur Überzeugung, in Lebensnähe $z u$ sein, oder religiös ausgedrückt zum Glauben an einen tragenden Lebensgrund.

Vor diesem Ereignis hat Janet jeglichen Kontakt zu einem Gegenüber

4 Hasenberg, Peter, "Ein Engel an meiner Tafel", in: film-dienst, Jahrgang 44, Heft 8, 1991, 21.

5 Eigene Übersetzung. 
vermieden, was im Film u.a. dadurch illustriert wird, dass Janet einen Spiegel in ihrem Zimmer zuhängt, um sich ohne Gegenüber den Blick auf sich selbst zu ersparen. Nach dem Wendepunkt in ihrem Leben wird Janet aufgrund eines Preises, den sie für eine Publikation gewinnt, aus dem Krankenhaus entlassen. Nun baut Janet wieder persönliche Kontakte auf, z.B. zum Schriftsteller Frank, zu ihrem ersten Liebhaber Bernard, zu ihrer Schwester und zum Leser ihrer Texte. Durch eine Therapie kommt Janet in Beziehung zu sich selbst und zu ihrer persönlichen $\mathrm{Ge}$ schichte und deckt die Diagnose Schizophrenie als falsch auf. So spielt sich Erlösung in „An Angel at My Table“ auf drei Ebenen ab - als Leben in Beziehung $z u$ einem tragenden Lebensgrund, in Beziehung $z u$ anderen Menschen und in Beziehung zum eigenen Selbst und zur persönlichen Geschichte.

Daneben hat aber Erlösung hier auch eine körperliche Dimension. Am Anfang des Films begegnet uns Janet als äußerst schüchternes Mädchen, das vor der Kamera wegrennt. Janets feuerrote Locken sind für sie als Kind und als Jugendliche ein Grund, sich unsicher und als Außenseiterin zu fühlen. Als junge Frau verneint Janet ihren Zyklus, ein deutliches Zeichen ihres Unwohlseins im eigenen Frauenkörper. Das Problem ihrer verfaulenden Zähne ignoriert Janet so lange wie möglich. Am Ende des Films findet Janet nicht nur zu einem Sein in Beziehungen, sondern auch zu körperlichem Selbstbewusstsein. Janet posiert nun selbstsicher für ein Pressefoto und durch ihre wachsende Sicherheit im eigenen Körper erscheint sie auch dem Zuschauer bei einem Bad im Ozean zum ersten Mal in körperlicher Schönheit. Ein einprägsames Bild körperlicher Erlösung zeigt der Film bei Janets Rückkehr von einem Europaaufenthalt. Janet stellt ihr Gepäck an einem neuseeländischen Strand $a b$ und blickt in aufrechter Haltung und mit erhobenen Armen aufs Meer. Campions Illustration von Erlösung ähnelt hier derjenigen in Lukas' Geschichte von der gekrümmten und geheilten Frau (Lk 13,10-13), indem beide Episoden Erlösung als körperlichen Zustand beschreiben. Wie Janet, so ist auch die gekrümmte Frau nach ihrer Heilung wieder fähig, aufrecht $z u$ stehen und mag in ihrem Lobpreis Gottes ähnlich wie Janet in diesem Moment ausgesehen haben.

\section{Sehnsucht nach Liebe - Gedanken zu "The Piano"}

Im Film „The Piano" geht es um eine Dreiecksbeziehung zwischen der stummen Schottin Ada, ihrem Mann Stewart und ihrem Liebhaber Baines, die beiden letzteren weiße Siedler in Neuseeland. Stewart, der die Heirat mit Ada über Adas Vater arrangiert hat, hat kein Verständnis für 
Adas enge Beziehung zu ihrem Piano, das sie als für sie lebensnotwendigen Stimmersatz von Schottland nach Neuseeland mitbringt. Stewart weigert sich, das Piano vom Strand in sein Haus zu transportieren und so ist die Beziehung zwischen ihm und Ada von Anfang an belastet. Baines dagegen begreift Adas Beziehung zum Piano instinktiv. Er erwirbt das Piano im Austausch gegen Land und hofft, durch einen weiteren Handel mit Ada seine erotischen Bedürfnisse zu erfüllen. Dieser Handel wird aber für Baines zunehmend unbefriedigend, weil er merkt, dass er zwar Adas körperliche Nähe, nicht aber ihre Liebe gewinnen kann. Nachdem er Ada das Piano zuriickgibt, wird Ada sich ihrer Liebe zu Baines bewusst. Stewart, der Adas und Baines' Liebe beobachtet, bemerkt nun selbst sein Bedürfnis nach Adas Liebe. Er bestraft Ada brutal für ihre Untreue, indem er ihr einen Finger abhackt. Danach lässt er sie aber mit Baines weggehen. ${ }^{6} \mathrm{Um}$ einerseits mit Baines glücklich zu sein und andererseits sich selbst zu entwickeln, muss Ada sich am Ende von ihrem Piano trennen, das zusehends zum Hindernis zwischen ihr und Baines wurde und ihr ein Überleben ohne die Entwicklung ihrer Sprachfähigkeit ermöglicht hatte. Auf der Fahrt über das Meer fordert Ada ihr Schicksal heraus und lässt sich von ihrem Piano in die Tiefe reißen. Nachdem sie sich einige Zeit willenlos sinken lässt, kämpft sie sich in der Tiefe des Meeres frei von der physischen und psychischen Bindung an ihr Klavier. Nach ihrem Auftauchen beginnt Ada ein neues Leben mit Baines, mit neu entdeckter Lust an der Sprache. Nach ihrem Kampf lebt Ada mit einem internalisierten Bild von sich am Meeresgrund weiter, das sie mit einem Gedichtzitat kommentiert: „There is a silence where hath been no sound, there is a silence where no sound may be. In the cold grave under the deep deep sea." (Es gibt ein Schweigen wo noch nie ein Laut war, es gibt ein Schweigen, wo nie ein Laut sein wird. Im kalten Grab unter dem tiefen, tiefen Meer. ${ }^{7}$ ) Das innere Bild ihrer selbst am Meeresgrund stellt für Ada eine Verbindung her zum Schweigen „unter dem tiefen Meer", zu dieser außerweltlichen, die Welt begründenden Realität. Dieses innere Bild wiegt Ada in den Schlaf, wie sie am Schluß aus dem Off erzählt; die Verbindung zum außerweltlichen Schweigen beruhigt sie. Als Bild der Verbindung zu einer die Welt begründenden Realität, die ihr sowohl Befreiung als auch Ruhe bringt, erinnert das Bild an das Gebet des

6 Die Wichtigkeit des Loslassens im Film "The Piano" wurde bereits erarbeitet von David Rhoads und Sandra Roberts in ihrem Beitrag "From Domination to Mutuality in The Piano and in the Gospel of Mark", in: Marsh, Clive und Ortiz, Gaye (Hg.), Explorations in Theology and Film, Oxford 1997. Allerdings erkennen die Autoren die Făhigkeit zum Loslassen nur in Ada und Baines und nicht in Stewart.

7 Eigene Übersetzung. 
Psalmisten: „Nur auf Gott zu ist still meine Seele, von ihm her ist meine Befreiung" (Ps 62,2).

Die Verwendung der Spiegelmetapher in Verbindung mit Adas Beziehung zu Baines zeigt, dass Ada die Beziehung zu einem Gegenüber braucht, um sich selbst neu zu sehen. Um ihre eigene verschüttete Sprache wiederzuentdecken und neu zu entwickeln, braucht Ada Baines als Partner, der sie beim Ausleben ihrer Liebe dazu ermutigt, zu flüstern und schon ihre Stimme erahnt. Die Wiederentdeckung ihrer Sprache bedeutet für Ada auch Wiedergewinnung der.Beziehung zu ihrer Umwelt, der sie sich durch ihre selbst auferlegte Stummheit jahrelang verweigert hatte. So ist Erlösung für Ada ein Sein in Beziehung zu Gott und ihrer Umwelt, durch das sie mehr sie selbst geworden ist.

Nicht nur Ada, sondern auch Stewart und Baines lernen im Film, dass sie kein selbstgenügsames Leben führen können, sondern auf Beziehung hin erschaffen sind. Stewart und Baines versuchen am Anfang des Films jeder auf seine Weise, ihr Leben unabhängig von ihrer Umwelt zu leben. Stewart versucht, durch das Roden des Busches den Kontakt mit seiner natürlichen Umgebung $\mathrm{zu}$ vermeiden und ein neues viktorianisches Schottland unabhängig von den Gegebenheiten in Neuseeland zu errichten. Baines hat für sich selbst ein Außenseiterdasein gewählt und gehört weder wirklich zur weißen Siedlergemeinschaft noch zu den Maoris, deren Tätowierungen er unvollständig auf seinem Gesicht trägt. Beide Männer lernen sich durch das Drama als Menschen kennen, die sich nach der Liebe eines anderen Menschen sehnen, ohne den sie sich als unvollständig empfinden. Die Spiegelmetapher kommt auch in Verbindung mit Stewart zum Einsatz. Noch vor seinem ersten Treffen mit Ada benutzt Stewart ein kleines verglastes Foto von Ada als Spiegel, in den er verstört blickt. In der Tat sieht Stewart sich selbst durch die Beziehung mit Ada neu. Allerdings lernt er durch Ada seine eigene Gewalttätigkeit kennen, die ihn zutiefst erschreckt.

Auch in "The Piano“ hat Erlösung eine körperliche Dimension. Der Film zeigt nicht nur die geistige, sondern auch die körperliche Schönheit der Beziehung zwischen Ada und Baines, unterstrichen durch bräunliches Licht und warme Farben. Als Erlöste sind Baines und Ada sowohl geistig als auch körperlich integre Menschen und sowohl geistig als auch körperlich in Beziehung. Adas langsames Freiwerden von den viktorianischen Moralvorstellungen findet seinen körperlichen Ausdruck in ihrer Befreiung von einzwängenden Kleiderschichten in der Beziehung mit Baines. ${ }^{8}$ Auch Stewarts Sehnsucht nach Adas Liebe hat sowohl eine

8 Diesem Aspekt des Films geht v.a. die englische Filmkritikerin Stella Bruzzi in ihrem Artikel „Bodyscape" nach, in: Sight and Sound, October 1993, 6-10. 
geistige als auch eine körperliche Dimension. Stewart sagt zu Ada gegen Ende des Films "I want to touch you" und seine Frustration entsteht, weil ihm sowohl eine geistige Beziehung als auch körperliche Berührung verwehrt bleiben. Wie wir sehen werden, ist das Motiv der Berïhrung auch im folgenden Film eine Kategorie von Erlösung.

\section{Sich berühren lassen und selbst berühren - Gedanken zu "The Portrait of a Lady"}

Der Film "The Portrait of a Lady" ist eine Verfilmung des gleichnamigen Romans von Henry James. ${ }^{9}$ Er handelt von der jungen Amerikanerin Isabel Archer, die als Waise zu ihrer in England lebenden Verwandtschaft zieht. Obwohl Isabel mehrere Verehrer hat, fühlt sie sich zu dem finanzschwachen aber ihr nobel erscheinenden Kunstsammler Osmond hingezogen, den sie in Florenz kennenlernt. Ihre Heirat mit Osmond gegen den Rat ihrer Verwandtschaft und Freunde markiert eine Veränderung in ihrem Charakter, die zeigt, wie sehr Isabel sich in Osmonds Persönlichkeit verschätzt hat. Die vormals äußerst lebendige und idealistische Isabel verschließt sich immer mehr in sich selbst und zeigt nach außen eine zwar makellose aber eiskalte Fassade. Nicht einmal der Vetter Ralph, mit dem Isabel vor der Heirat durch eine ehrliche Freundschaft verbunden war, kann die Fassade durchbrechen. Osmond selbst löst jedoch in Isabel eine Wende aus, als er ihr im Zusammenhang mit einem Streit ein Bein stellt und Isabel fällt. Die englische Filmkritikerin Lizzie Francke weist auf die Wichtigkeit dieses Moments hin, wenn sie ihn als "einen der kühnsten, schockierendsten Momente" des Films bezeichnet. ${ }^{10}$ Am Anfang des Films erklärte Isabel „I shall never crawl“, nun ist sie genau in der Lage, in die sie nie kommen wollte. Nach diesem Ereignis beginnt Isabel, Schritt für Schritt ihre perfekte Fassade zu demontieren. Als der todkranke Ralph nach England zurückkehrt, nutzt

9 Der Film erhielt v.a. im deutschsprachigen Raum negative Kritik. Er ist zugegebenermaßen anfangs schwer verständlich. Durch eine ausführlichere Beschäftigung mit ihm kann man jedoch die reiche metaphorische Sprache des Films schätzen lernen. Ich finde es schade, dass Feldvoß und vor allem Lachat, der sich an Nebensächlichkeiten wie der Frisur von Nicole Kidman in "The Portrait" und derjenigen von Kerry Fox in "An Angel" aufhält, die für das Verständnis des Films so wichtigen Metaphern völlig außer Acht lassen. Vgl. Feldvoß, Marli, "Portrait of a Lady“, in: epd Film, Heft 1, 1997, 40-41 und Lachat, Pierre, "Das aufgeputzte Bauernmädel", in: Filmbulletin, Jahrgang 38, Heft 209, Dezember 1996, 11-13.

10 Francke, Lizzie, "On the brink", in: Sight and Sound, November 1996, 9, eigene tibersetzung. 
Isabel dies, um aus dem Ehegefängnis auszubrechen. Sie folgt Ralph an sein Sterbelager in England und kann dort zum ersten Mal Ralph und sich selbst eingestehen, in ihrer Heirat mit Osmond einen Fehler begangen zu haben. In ihrem Zusammensein werden Ralph und Isabel einer sie beide umgreifenden Realität gewahr. "Why should there be pain? It's not the deepest thing", fragt Isabel, offensichtlich überzeugt, dass es eine Wirklichkeit gibt, die der tragende Grund selbst einer schmerzhaften Erfahrung ist.

In einer an 1 Kor 13,8 erinnernden Antwort gibt Ralph dieser Wirklichkeit einen Namen: „Pain is deep, but it passes ? Love remains." In ihrer gegenseitigen Liebe fühlen sich Ralph und Isabel verbunden mit einem umfassenden, alles tragenden Grund, den sie Liebe nennen. Wie in den vorhergehenden Filmen, so drückt auch hier die Spiegelmetapher aus, dass Isabel zu ihrer eigenen Selbstwerdung die Beziehung zu einem Gegenüber braucht. Isabel blickt während des ganzen Films in mehrere Spiegel. Diese Spiegelbilder tauchen immer im Zusammenhang mit menschlichen Beziehungen auf. Bei der letzten Begegnung zwischen Isabel und Ralph wird die Metapher nochmals aufgegriffen und gleichzeitig erweitert. Ralphs und Isabels Gesichter sind sich direkt gegenüber, ein bildlicher Ausdruck dafür, dass Isabel sich durch Ralphs Liebe besser selbst sehen kann, dass sie sich selbst mit ihren Fehlern annehmen kann und diese nicht mehr hinter einer Fassade verstecken muss. Dieses letzte "Spiegelbild“ erinnert noch einmal an 1 Kor 13: „Jetzt schauen wir in einen Spiegel und sehen nur rätselhafte Umrisse, dann aber schauen wir von Angesicht zu Angesicht. Jetzt erkenne ich unvollkommen, dann aber werde ich durch und durch erkennen, so wie ich auch durch und durch erkannt worden bin" (1 Kor 13,12). Ralph, der Isabel während des ganzen Films wie ein Gewissen folgt und immer wieder versucht, ihre Fassade zu durchbrechen, erkennt Isabels Wesen intuitiv, „from the voices of the air", wie er es selbst ausdrückt. Dieses Erkennen ermöglicht es Isabel, sich selbst zu erkennen und anzuerkennen. Isabel scheint das erreicht $\mathrm{zu}$ haben, was junge Frauen in Campions persönlicher Einleitung zu Henry James' Roman in einem Gespräch über die Liebe ausdrückt und was gleichzeitig als Beschreibung von Erlösung für alle hier behandelten Filme gelten kann: „It means finding a mirror, the clearest and the most loyal mirror. "11 Erlösung ist also für Isabel ein Sein in Beziehung zu einer alles umgreifenden Liebe, die sich als Liebe zu einem Menschen und dadurch als erneuerte Liebe zu sich selbst zeigt.

Wie schon angedeutet, spielt das Motiv der Berührung als

11 Lizzie Francke sieht die ersten Einstellungen des Films im modemen Australien als Campions eigenes Vorwort zu Henry James' Roman, vgl. Francke, November 1996, 6. 
körperlicher Ausdruck von Beziehung und damit verbunden das Motiv der Hand eine wesentliche Rolle in "The Portrait of a Lady". Die Hand wird als Motiv bereits am Anfang eingeführt: Der Filmtitel erscheint auf eine Frauenhand geschrieben. Was die Motive „Beriihrung“ und „Hand" anbetrifft, oszilliert der Film in der Spannung zwischen Berührung innerhalb viktorianischer Etikette und Berührung als Ausdruck wirklicher Beziehung. Vor ihrer Heirat mit Osmond ist Isabel zwar in Berührung, aber nur auf passive Art. Ganz viktorianisch hält Isabel ihre Hand für Handküsse hin. In einer Fantasie, in der mehrere Verehrer gleichzeitig auftauchen, stellt Isabel sich als von den Händen der Verehrer Berührte vor. Bei beiden Arten der Berührung sind die Männer die aktiv berührenden, während Isabel passive Empfängerin der Berihhrungen ist. Während ihrer Ehe mit Osmond zieht Isabel sich nicht nur vom geistigen Kontakt mit ihrer Umwelt zurück, sondern auch von körperlicher Berïhrung. Sie trägt Handschuhe und oft einen Schleier über dem Gesicht. Bei ihrem letzten Zusammentreffen mit Ralph findet Isabel aber zu einer anderen Art von Berührung. Isabel berührt zum ersten Mal selbst aktiv und wartet nicht mehr passiv auf Berührung. So wie die geistige Beziehung zu Ralph für sie erlösend ist, so ist auch ihre neu entdeckte Fähigkeit zu berühren als körperliches Zeichen ihres geistigen Verbundenseins erlösend. Im Zusammenhang mit der erlösenden Wirkung von Berïhrung stellen sich zahlreiche biblische Assoziationen ein, vor allem die Heilungsgeschichten des Neuen Testaments. Am meisten scheint Isabels Erlöstsein mit der Heilung der blutflüssigen Frau gemeinsam zu haben (Mk 5,25-34). Beide Frauen finden ihr Heil, indem sie entgegen der gesellschaftlichen Konvention aktiv berühren.

\section{Erlösung in den Augen der Theologie am Ende des 20. Jahrhunderts}

Zwischen Campions Verständnis von Erlösung und der Auffassung von Theologen des 20. Jahrhunderts gibt es einige Übereinstimmungen. Haben Theologen Erlösung über weite Strecken der Theologiegeschichte oft einseitig als Geschehen zwischen Gott und Mensch betrachtet, so unterstreichen Theologen am Ende des 20. Jahrhunderts neben dieser individuellen Ebene auch eine soziale Ebene von Erlösung. Die Lehre von der Trinität beschreibt Gott selbst als Beziehung. Die Schöpfung und der Mensch in ihr sind deshalb als Abbild Gottes ebenfalls auf Beziehung hin angelegt. Erlösung bedeutet also für Menschen in der Theologie ebenso wie in den Filmen Campions, in Beziehung zu sein, „relational“ zu sein. ${ }^{12}$

12 Gisbert Greshake verwendet dieses Adverb als Beschreibung von Erlösung in „Erlöst in einer unerlösten Welt?", Mainz 1987. 
Erlösung betrifft, ähnlich wie in Campions Filmen, drei Ebenen menschlichen Daseins. Auf der Ebene Gott - Mensch bedeutet Erlösung, sich selbst als begrenzt und gleichzeitig in einer unbegrenzten Realität gegründet zu empfinden. Friedrich Schleiermacher beschreibt dieses $\mathrm{Be}$ wusstsein als "das unmittelbare Bewusstsein von dem allgemeinen Sein alles Endlichen im Unendlichen und durch das Unendliche". ${ }^{13}$ Eine erlöste Person ist sich eines allumfassenden Grundes der Welt bewusst. Wie wir gesehen haben, drückt sich dieses Bewusstsein in „An Angel at My Table" als Gewahrwerden eines tragenden Lebensgrundes, in "The Piano" als Erfahrung eines ewigen Schweigens und in "The Portrait of a Lady" als Bewusstsein einer selbst Schmerz umfassenden Liebe aus.

Auf der Ebene Mensch - Welt bedeutet Erlösung ein In-BeziehungSein des Menschen zu den Mitmenschen und zur Natur. Weil Gott, wie Greshake meint, in der Schöpfung verleiblicht ist, ist Gemeinschaft mit der Schöpfung und mit den Mitmenschen verleiblichte Gemeinschaft mit Gott. Die Beziehung des Menschen zur Schöpfung und zum Mitmenschen wird "Medium, Ort und Symbol der Beziehung zu Gott". ${ }^{14}$ In ähnlicher Weise werden, wie beschrieben, in den Filmen die Beziehungen zum Lebensgrund bzw. zum ewigen Schweigen und zur umfassenden Liebe jeweils durch wieder hergestellte soziale Beziehungen der Hauptfiguren komplementiert. Die Beziehungen der Hauptfiguren zum tragenden Grund verwirklichen sich gleichsam in deren Beziehungen zu Umwelt und Mitmenschen.

Auf der Ebene des Individuums bedeutet Erlösung ein integres Personsein, das die eigenen Stärken entwickeln und die eigenen Schwächen annehmen kann. Wahre menschliche Gemeinschaft kommt nur zwischen freien Partnern zustande. "Liebe setzt den Selbstand des anderen voraus", 15 schreibt Greshake. Die individuelle Ebene und die Ebene Mensch - Welt bedingen sich gegenseitig. Einerseits können Individuen nur durch die Gemeinschaft existieren. Eltern, Familie und Gesellschaft sind essentiell für die Subjektwerdung des Einzelnen. Andererseits existiert die Gesellschaft nur, wenn einzelne Individuen sie bilden. Erlösung manifestiert sich dann in einem gelungenen Austausch zwischen dem Individuum und der Gemeinschaft: „Da ich also in der Communio ,Ich' bin, es bleibe, ja es mehr und mehr werde, kann ich der Gemeinschaft auch das Beste geben, was ich habe: mich selbst" ${ }^{16}$ In den Filmen drückt gerade die Spiegelmetapher diesen Austausch zwischen dem Individuum und

13 Schleiermacher, Friedrich, Über die Religion, Berlin 1995, 53.

14 Greshake 1987, 36.

15 Ders., 37.

16 Ders., 47. 
den Mitmenschen aus. In der Beziehung zum Spiegelbild sind die Beziehung zu sich selbst und die Beziehung zum Gegenüber untrennbar miteinander verbunden.

Allerdings gibt es auch Unterschiede zwischen Campions Erlösungsverständnis und dem Verständnis der Theologie. Diese Unterschiede können der Theologie zur Anregung werden.

\section{Für ein erweitertes Verständnis von Erlösung}

Ein Bereich, in dem Theologie von den Filmen Campions lernen kann, ist deren Verständnis von Erlösung als körperlichem und geistigem Zustand. Wie wir gesehen haben, wird Erlösung durch Bilder körperlicher Integrität dargestellt wie $2 . B$. diejenigen von Janets schönem Körper im Ozean oder ihrer aufrechten, Selbstvertrauen ausstrahlenden Geste mit erhobenen Armen. Für Ada macht Erlösung nicht nur eine spirituelle, bisher unentwickelte Seite sichtbar, sondern auch die Schönheit ihres Körpers, die bisher unter einzwängenden Kleiderschichten verborgen war. Wenn Adas und Baines Liebe erlösend ist, so ist es nicht nur die spirituelle Seite dieser Liebe, sondern auch die Sexualität und Erotik, die sie zu integren Menschen macht. In "The Portrait of a Lady" wird Erlösung als Fähigkeit zur Berührung beschrieben. Darüber hinaus stimme ich der englischen Filmkritikerin Stella Bruzzi völlig zu, wenn sie den Kamerastil in "The Piano" als einen der Berührung und nicht so sehr des Blickes auf Objekte vor der Kamera bezeichnet; ein Stil, der auch in den anderen hier behandelten Filmen augenscheinlich ist. ${ }^{17}$ Nahaufnahmen wie die von Baines' dreckigen Fingernägeln, dem Blumenmuster auf dem Kleid von Janets Mutter und Isabels Gesicht wurden von mehreren Kritikern als Stil beschrieben, der Campion auszeichnet. ${ }^{18}$ Campion macht diesen Stil noch ausdrücklicher, indem sie die Nähe der Berührung mit Bildern der Distanz des Blickes kontrastiert: ein Moment der nicht funktionierenden Beziehung in "The Piano" ist derjenige, in dem Stewart Ada durch den Sucher einer Kamera beobachtet. In „The Portrait" wird die fehlende Nähe zwischen einem Verehrer und Isabel spürbar, wenn dieser Isabel besucht, um sie nach eigener Aussage „anzusehen“. Die Filmkritikerinnen Ursula Vossen und Stella Bruzzi identifizieren die Distanz des

17 Bruzzi, Stella, "Tempestuous petticoats: costume and desire in The Piano", in: Screen, Jahrgang 36, Heft 3, Autumn 1995, 265.

18 Z.B. Ober, Juan-Ramón García, "Ein Engel an meiner Tafel“, in: medien praktisch, Heft 3, 1991, 37. 
Blicks als männlich und Campions Nähe der Berührung als weiblich. ${ }^{19}$ Durch diese Nahaufnahmen minimiert Campion die Distanz zwischen dem Zuschauer und dem gefilmten Objekt, so dass nicht nur die Charaktere in den Filmen, sondern auch die Zuschauer die Geschichte mehr berühren als sehen, dass eine körperliche Erfahrung möglich wird.

Campions Betonung auf der Verbindung zwischen körperlicher und spiritueller Erfahrung, die durch ihren Kamerastil und ihr Verständnis von Erlösung offensichtlich wird, ist nicht neu. Auch wenn zwischen ihren Filmen und den Texten des Alten und des Neuen Testaments im Hinblick auf ihren gesellschaftlichen Rahmen und ihren theologischen Inhalt selbstverständlich erhebliche Unterschiede bestehen, so gibt es doch eine Ähnlichkeit in der Art und Weise, wie Körper und Geist jeweils in Beziehung gesetzt werden. Im Alten Testament drückt sich eine enge Beziehung zwischen Gott und Mensch auch körperlich aus. Jahwe berührt z.B. die Lippen des Propheten (Jer 1,9), er ergreift bei der Hand $(J e s \quad 42,6)$ und schreibt einen Menschen in seine eigene Hand ein (Jes $49,16)$. Wenn das Neue Testament Jesus in vielen Geschichten darstellt als jemand, der Menschen heilte, so weist es damit auf die Verbindung von Körper und Geist hin. Diese Geschichten zeigen, dass das Reich Gottes, das in Jesus beginnt, sowohl eine körperliche, als auch eine spirituelle Wirklichkeit ist. Die durch Jesus gestiftete Erlösung bewirkt im Neuen Testament das geistige und das körperliche Heilsein des Menschen. So wie Campion will, dass wir ihre Filme nicht nur hören und sehen, sondern auch berühren, so unterstreicht der erste Johannesbrief die Wichtigkeit nicht nur des Hörens und Sehens, sondern der Berührung des Wortes des Lebens mit den eigenen Händen (1 Joh 1,1).

Leider ist der körperliche Aspekt von Erlösung durch die Theologiegeschichte hindurch zu einem großen Teil verloren gegangen. Selbst Greshake, der die Verbindung zwischen Erlösung und menschlichen Beziehungen zwar plausibel erklärt hat, schafft es nicht, den körperlichen Aspekt von Erlösung in sein Konzept einzubeziehen. Obwohl er wiederholt das Wort „Verleiblichung" verwendet, schreibt er doch von der spirituellen Seite der menschlichen Person und menschlicher Beziehungen. Nachdem er erklärt, wie Erlösung eine Wirklichkeit des Geistes, nicht aber eine Wirklichkeit des Körpers wird, muss konstatiert werden, dass das Wort "Verleiblichung" hier nicht in seiner ursprünglichen Bedeutung verwendet wird. Bei einem solchen Konzept von Erlösung bleibt ein wesentlicher Teil des Menschen permanent außen vor und deshalb unerlöst.

19 Bruzzi 1995, 265; Vossen, Ursula, "Wie ein klarer Spiegel", in: film dienst, Jahrgang 50, Heft 1, 1997, 10. 
Mary Grey, die zwei Jahre nach Greshake sehr ähnliche Gedanken zum Thema Erlösung entwickelt hat, geht in ihrem abschließenden Kapitel einen Schritt weiter, indem sie Erotik und Sexualität als Kategorien von Erlösung bezeichnet. ${ }^{20} \mathrm{Ihr}$ Buch macht deutlich, dass die körperliche Seite der Erlösung wieder mehr ins Herz der Theologie gerückt werden muss.

Campions Filme können dazu anregen, dies zu tun. Weil Gott den Menschen als Geist und Körper erschaffen hat, muss Erlösung den Körper einbeziehen, will sie den ganzen Menschen betreffen. Die Theologie sollte körperliche Erfahrungen in ihrer Lehre von der Erlösung berücksichtigen. Sich im eigenen Körper wohlzufühlen und ihn zu mögen, auch wenn er rote Haare hat, das macht Erlösung in Janets Leben aus. Im Blick auf Adas und Baines Erfahrung von Erlösung könnte Theologie fragen, ob Erotik und Sexualität nicht eine Erfahrung der Communio mit Gott durch absolute Einheit mit einem anderen Menschen sein kann. "The Portrait of a Lady“ betrachtet Relationalität als spirituellen und körperlichen Zustand. Bedenkenswert für die Theologie wäre hier, dass Menschen einander dann im vollen Sinne anerkennen können, wenn wir nicht nur geistig, sondern auch körperlich miteinander in Berührung und in gleichberechtigter Beziehung sind.

20 Grey, Mary, Redeeming the dream: feminism, redemption and Christian tradition, London 1989, 157-165. 